\title{
Regional Anesthesia Techniques for Pain Management for Laparoscopic Surgery: a Review of the Current Literature
}

\author{
Alvaro Andrés Macías ${ }^{1}$ (D) . John J. Finneran² \\ Accepted: 3 January 2022 / Published online: 27 January 2022 \\ (c) The Author(s), under exclusive licence to Springer Science+Business Media, LLC, part of Springer Nature 2022
}

\begin{abstract}
Purpose of Review The field of regional anesthesia has evolved tremendously in the last 15 years. New anesthesia protocols for ambulatory surgery and enhanced recovery after surgery have been developed as well. The focus of these techniques and protocols has centered on patient satisfaction and pain control while minimizing the use of opioids. The field of ambulatory surgery and anesthesia continues to evolve, and regional anesthesia and its plane techniques are at the center of these changes. Recent Findings Recent research has shown that regional techniques contribute to better pain control and patient experience and may decrease patient readmission rates. The safety of these techniques has been validated when performed by experienced practitioners. New techniques such as the erector spinae block (ESP) have been studied in the setting of laparoscopic surgery with promising results.

Summary Regional anesthesia techniques for patients presenting for laparoscopic surgery are safe and seem to provide benefits. Those are related to patient experience, pain control, and readmission rates. Different techniques can be applied to a specific type of intervention. Application of these techniques depend on the clinical picture and patient. Future research may help us clarify how these techniques may improve patient satisfaction and operating room efficiency. New regional blocks may also develop based on what we know today.
\end{abstract}

Keywords Ambulatory $\cdot$ Anesthesia $\cdot$ Regional pain $\cdot$ Laparoscopic surgery

\section{Introduction}

Following nausea and vomiting, pain is the most common reason for hospital admission after laparoscopic surgery [1•]. Pain associated with laparoscopic surgery is multifactorial, including visceral, incisional, and referred pain components. Visceral pain is a complex condition that can be caused by mechanical traction, dilation, spasm, inflammation, ischemia, and chemical stimulation [2]. Peripheral regional anesthesia techniques for laparoscopic surgery mainly focus on management of incisional rather than visceral pain. Thus, patients with moderate to severe acute visceral pain (e.g.,

This article is part of the Topical Collection on Acute Pain Medicine

Alvaro Andrés Macías

amacias@bwh.harvard.edu

1 Massachusetts Eye and Ear, Brigham and Women's Hospital, Harvard Medical School, Boston, MA, USA

2 University of California San Diego, La Jolla, CA, USA patients with acute cholecystitis or following more invasive laparoscopic surgeries such as bowel resection) may benefit more from neuraxial rather than peripheral regional anesthesia techniques (i.e., epidural, paravertebral, and intrathecal blocks). Recent regional techniques have been developed to address visceral pain while avoiding the potential complications associated with neuraxial techniques such as bleeding, infection, and spinal cord injury. These "more midline" peripheral block techniques (e.g., erector spinae plane and quadratus lumborum blocks) may address both visceral and incisional pain.

The development of enhanced recovery after surgery (ERAS) protocols and robotic techniques [3] for laparoscopic surgery have propelled regional techniques to a new prominence. Clinicians are seeking novel modalities to decrease post-operative opioid requirements and their associated side effects such as nausea and decreased bowel motility. These adverse effects delay recovery after surgery, increasing length of stay, and costs to the health care system while worsening patient satisfaction [4]. 


\section{Trends in Ambulatory Surgery}

Ambulatory surgery has changed significantly in the last quarter century $[1 \bullet]$. The number of ambulatory surgeries has increased steadily and surgeries that once required hospital admission can now frequently be performed in an ambulatory setting. For example, $60 \%$ of cholecystectomies are now performed in ambulatory surgery centers [5]. It is expected this number will increase in the coming years, and this trend may be hastened as a result of the COVID-19 pandemic. Some authors have gone as far as suggesting that ambulatory appendectomy could become the standard of care [6]. It is not only new surgical approaches that have made these trends possible, but also new anesthetic drugs and regional anesthesia techniques. Nausea, vomiting, and pain are frequently the greatest impediment to ambulatory surgery. Thus, addressing pain with non-opioid medications and regional anesthetic techniques may provide better pain control while minimizing the undesirable side effects associated with opioid analgesics and, thereby, facilitate surgery in an ambulatory surgery center setting $[1 \bullet]$.

\section{Laparoscopic Surgery Techniques}

It is important to consider the continuing advances in surgical techniques when contemplating regional anesthesia techniques for laparoscopic surgery. Traditional laparoscopic surgery is based on the concept of triangulation, which facilitates organ exposure and ergonomics. Over the last 20 years, clinicians and patients have experienced its benefits. However, new trends in outpatient surgery have created demand for anesthetics tailored to laparoscopic surgery. For example, single-incision laparoscopic surgery (SILS) [7] has been recently used for several common procedures such as appendectomy and cholecystectomy. SILC involves a $2-\mathrm{cm}$ incision into the umbilicus between the T7 and T11 intercostal nerves [8]. This evolving technique will likely require more evidence prior to its wide utilization. However, the apparent benefits include reduced pain, risk of bleeding, surgical site infection, visceral injury, and port site herniation. Given the potential reduction in pain associated with SILS, anesthetic techniques will need to be further refined for these surgeries, should they achieve widespread utilization.

\section{Pain following Laparoscopic Surgery}

Laparoscopic surgery has numerous perceived advantages over open surgical procedures, including reduction in postoperative pain and opioid requirements due to smaller surgical incisions, less impairment of pulmonary function resulting from splinting, decreased incidence of ileus and respiratory depression, and to reduction in the potential for prolonged wound healing and wound dehiscence [9]. Nevertheless, there is still significant potential for pain following laparoscopic surgery. Injury to the skin, abdominal musculature, peritoneal lining, and viscera may contribute to this pain. The anatomy of the peritoneum is complex and must be understood for a proper understanding of the pain associated with laparoscopic surgery. The parietal and visceral peritoneal linings have distinct embryologic origins and, therefore, differing innervation. The parietal peritoneum derives from the somatopleural layer and, thus, is innervated by the associated dermatomes [9]. Conversely, the visceral peritoneum is derived from the splanchnopleural layer and is innervated by the autonomic nerves supplying the visceral organs. The differing embryologic origins of the parietal and visceral layers of the peritoneum give rise to distinct pain sensations.

Painful surgical stimuli exert their effects not only by primary noxious stimulus to the central nervous system, but also by initiating positive feedback cell signaling loops in the dorsal horn of the spinal cord inducing a state of hypersensitivity to pain. As a result of this self-amplifying cell signaling cascade, pain experienced following laparoscopic surgery may last considerably longer than the primary painful stimulus. These changes that occur in the central nervous system make the case for preoperative and thus preemptive interventions to manage pain before an inciting painful stimulus. Preoperative administration of local anesthetics may attenuate or completely prevent these hyperalgesic changes in the central nervous system. Indeed, there is evidence that preoperative nerve blocks provide superior analgesia during the early postoperative period compared to the same blocks administered immediately postoperatively [10].

While laparoscopic surgery produces less trauma to the skin and abdominal wall musculature, the pain resulting from peritoneal stretching, burning, muscle spasm, inflammation, ischemia, and chemical stimulation can be severe and require significant opioid administration. In fact, the early (first $24 \mathrm{~h}$ ) pain and opioid requirements following laparoscopic surgery may be greater than those following open laparotomy [11]. However, after this immediate postoperative phase, the relationship is reversed, with pain worse in patients who underwent laparotomy. The varied sources and types of pain produced by laparoscopic surgery call for a multimodal approach to analgesia flowing these surgical procedures [12]. And, the more marked early pain associated with laparoscopic surgery would seem to suggest that single-injection peripheral nerve blocks with a long-acting local anesthetic (i.e., bupivacaine) may alleviate much of the severe postoperative pain after laparoscopic surgery and facilitate same-day discharge. 


\section{Anatomy of the Abdominal Wall}

Understanding the anatomy of the abdominal wall [13, 14•] is essential for a regional anesthesiologist employing techniques that can be of used to provide analgesia following laparoscopic surgery. The anatomy of the abdominal wall consists of multiple layers of muscle; each of which is surrounded by its own fascia $[15,16]$. Various muscles make up the abdominal wall: Lateral to the vertebral column, the quadratus lumborum, psoas major, and iliacus muscles reinforce the posterior aspect of the wall. The caudal ends of the psoas major and iliacus muscles pass into the thigh and are major flexors of the hip joint. There are 4 paired muscles of the anterior and lateral abdominal wall [13]: the anterior rectus abdominis muscles, and from deep to superficial, the 3 lateral muscles: transversus abdominis, internal oblique, and external oblique muscles. In the lateral abdomen, the three bellies of the oblique muscles, transversus abdominis, internal oblique, and external oblique, overlie one another. Medially, these three muscles insert into the aponeurotic linea semilunaris just lateral to the rectus abdominis muscle. The shape and location of the muscular bellies and the linea semilunaris help identify the layers which are the main reference for the different variations of transverse abdominis plane (TAP) blocks. Anteriorly, the rectus abdominis spans the distance between the thoracic wall (superiorly) and the pelvis (inferiorly). Structural continuity between posterior, lateral, and anterior regions of the abdominal wall is provided by thick fascia posteriorly and by flat tendinous sheets (aponeuroses) derived from muscles of the lateral wall. A fascial layer of varying thickness separates the abdominal wall from the peritoneum, which lines the abdominal cavity.

The thoracolumbar fascia has been the target of several cadaveric studies for peripheral regional anesthetics and has been suggested responsible for the spread of local anesthetic injected in certain "plane" blocks into the paravertebral space [15]. It is comprised of three layers: anterior, middle, and posterior fascia, and is wrapped around the erector spinae and quadratus lumborum muscles. The anterior layer of this fascia is located posterior to the erector spinae and is also the initial aponeurosis of the latissimus dorsi. The middle layer separates the erector spinae and the quadratus lumborum muscles. While the middle and anterior layers meet at the lateral border of the erector spinae, the posterior layer covers the anterior boundary of the quadratus lumborum. The three layers of fascia converge at the lateral border of the quadratus lumborum to form the initial aponeurosis of the internal abdominal oblique and transversus abdominis muscles. It is believed that the thoracolumbar fascia acts as a pathway for local anesthetic spreading to the paravertebral space, and for the distribution of the spinal nerve branches (the lateral branches of posterior branch of L1-L3 spinal nerve) and sympathetic fibers [17].

The innervation of the abdominal wall is derived from anterior divisions of the thoracolumbar spinal nerve roots [13]. The T6 to T11 spinal roots give rise to the intercostal nerves and $\mathrm{T} 12$ to the subcostal nerve. The $\mathrm{L} 1$ spinal nerve root becomes the iliohypogastric and ilioinguinal nerves. The T6 dermatome is a small area below the xyphoid. The T7 and T8 nerves pass toward the xyphoid, parallel to the costal margin. Anterior intercostal nerve branches arising from T6 to T8 form beneath the rectus muscle and traverse variable distances between the posterior rectus sheath and the transversus abdominis muscle in the TAP before penetrating anteriorly through the rectus sheath. After a further course between the rectus sheath and rectus muscle, they pass into the muscle. However, there are exemptions where $\mathrm{T} 6$ to $\mathrm{T} 8$ nerves may pass directly into the rectus muscle near the costal margin. In this anatomic variant a block placed between the rectus abdominis muscle and the posterior rectus sheath close to the midline may miss these nerves $[17,18 \bullet]$. Each segmental nerve has a lateral branch that leaves the main nerve posteriorly, near the angle of the rib, and passes with it a short distance. The lateral branch then emerges obliquely through the overlying muscles around the midaxillary line. These branches arise before the nerve enters the TAP, although the T11 and T12 lateral branches may have a short course within or through the TAP. The ilioinguinal and iliohypogastric nerves have a different course than the thoracic nerves. These nerves remain deep to the transversus abdominis muscle until the middle one-third of the iliac crest (measured from anterior superior iliac spine to posterior superior iliac spine); anterior to this, they are usually found in the TAP.

Due to the rich vascular supply to the abdominal wall a TAP, and other abdominal plane blocks commonly produce local anesthetic absorption comparable to infraclavicular and axillary brachial plexus blocks. For these techniques, ropivacaine usually reaches a peak plasma level in a mean time of $25 \mathrm{~min}$. This is much slower than epidural and interscalene blocks, with ropivacaine reaching peak plasma levels in less than $20 \mathrm{~min}$ [13].

\section{Regional Techniques}

Regional anesthetics for abdominopelvic surgeries fall broadly into two categories, neuraxial blocks and peripheral blocks. Neuraxial blocks include intrathecal or epidural administration of opioid or local anesthetic and paravertebral blocks with local anesthetic. Although individual 
nerves have been targeted for abdominal regional anesthesia, peripheral blocks of the abdominal and pelvic analgesia usually are generally "plane blocks." Instead of injecting local anesthetic directly around a target nerve as with conventional peripheral nerve blocks, plane blocks utilize a large volume of more dilute local anesthetic to infiltrate a plane the targeted nerves traverse.

Neuraxial blocks can produce surgical anesthesia and dense postoperative analgesia. However, bupivacaine and ropivacaine delivered to the intrathecal and epidural spaces have a relatively short duration of action, measured in minutes when administered intrathecally as opposed to hours for perineural administration $[19,20]$. Consequently, epidural or intrathecal local anesthetics must be administered by continuous infusion via an indwelling catheter when utilized for postoperative analgesia. While thoracic epidural infusions provide outstanding analgesia following laparoscopic surgery, there are numerous disadvantages to epidural analgesia [21, 22]. Chiefly among these is the requirement for inpatient admission and monitoring precluding the use of this modality in ambulatory surgery. Additionally, epidural analgesia is associated with risks that include profound hypotension, epidural abscess, and hematoma resulting in spinal cord compression. For these reasons, thoracic epidural analgesia is ideally utilized for laparoscopic surgeries that will themselves necessitate hospital admission (e.g., laparoscopic colorectal resections). For major laparoscopic colorectal surgery, thoracic epidural analgesia may decrease not only pain and opioid consumption, but also the time to gastrointestinal recovery, while improving tolerance of oral intake $[22,23]$.

The presence of opioid receptors in the spinal cord allows for intrathecally or epidurally administered opioids to exert a local effect at the level of the spinal cord [24]. This produces dense analgesia while limiting the deleterious effects of systemic opioid administration. In contrast, opioid receptors are not found in the peripheral nervous system. Nevertheless, studies comparing the addition of opioids to local anesthetic based peripheral nerve blocks have found mixed results [25]. Some authors have shown benefit to addition of opioids to peripheral nerve blocks, while others have demonstrated no benefit beyond what is observed with systemic administration [26-28]. Morphine is the most commonly used opioid for intrathecal administration due to its prolonged effect compared to more lipophilic shorter acting opioids such as fentanyl. Intrathecally administered morphine, which may be administered in combination with local anesthetic or alone, decreases pain and reduces the need for systemic opioid therapy following laparoscopic surgery [29, 30]. However, as with systemic administration, intrathecally administered opioids produce respiratory depression in a dose dependent manner. Intrathecal morphine is also associated with other opioid related side effects, such as nausea, vomiting, and pruritus [31]. Morphine administered intrathecally exhibits a bimodal pattern of respiratory depression, with an early phase (approximately $2 \mathrm{~h}$ following administration) and a late phase (usually $6-12 \mathrm{~h}$ but may be as late as $24 \mathrm{~h}$ ). The late phase of respiratory depression is the result of rostral spread of the hydrophilic morphine molecules within the cerebrospinal fluid to the respiratory drive centers of the brain [32]. Thus, patients require monitoring for approximately $24 \mathrm{~h}$ following surgery. This makes intrathecal morphine an excellent analgesic option for large surgical procedures necessitating hospital admission but precludes its use for ambulatory surgeries. When used as part of a multimodal ERAS protocol, intrathecal morphine may also facilitate earlier discharge following large laparoscopic surgeries [29].

Paravertebral blocks bridge the gap, anatomically and metaphorically, between neuraxial and peripheral regional anesthesia. These blocks target local anesthetic lateral to the epidural space where the spinal nerve roots emerge. Paravertebral blocks, in contrast to local anesthetic injections in the intrathecal or epidural spaces, have a duration of action that may be well matched to the pain following many laparoscopic surgeries. Thoracic paravertebral blocks, with or without ultrasound guidance, have been shown to decrease both pain scores and opioid consumption in the first $24 \mathrm{~h}$ following laparoscopic cholecystectomy [10, 33, 34]. Unfortunately, paravertebral blocks often require multiple bilateral injections as there is generally only a 1-2 dermatome level spread with each injection [35]. Each of these injections is associated with approximately a $0.3-0.5 \%$ risk of pneumothorax [36, 37]. Thoracic paravertebral blocks are also associated with profound sympathectomy, as the sympathetic innervation to both the heart and splanchnic vasculature are anesthetized. Thus, there is great need for more peripheral regional anesthetic techniques that are appropriate for use in an ambulatory setting and have limited side effects and risks.

To facilitate both regional anesthesia education as well as research in this developing field, agreement and uniformity in the nomenclature and terms of different peripheral regional blocks is of great importance. Recent expert consensus have been published with the intention of unifying concepts and definitions [14•]. Of particular importance in studying these emerging techniques, research publications have often differed in their technique descriptions when referring to the same block by name. The clinical implications of these differences are significant as the injected local anesthetics will produce varied results by targeting different nerves and by their spread across the abdominal wall. We have summarized the clinical applications of these new blocks in Table 1 . The following terms are now generally accepted for the most common abdominal wall plane and peripheral nerve blocks of the abdomen: 
Table 1 Regional anesthetics for common laparoscopic surgeries

\begin{tabular}{ll}
\hline Surgical procedure & Blocks \\
\hline Ventral hernia repair & Paravertebral (T7-T11), rectus sheath \\
Cholecystectomy & Subcostal transversus abdominis plane, Erector spinae plane, paravertebral \\
Single-incision laparoscopic surgery & Rectus sheath, paravertebral (T9-T11) \\
Inguinal hernia repair & Transversus abdominis plane, paravertebral (T9-T11), ilioinguinal, and iliohypogastric \\
Colorectal surgeries & $\begin{array}{l}\text { Paravertebral, erector spinae plane, quadratus lumborum, thoracic epidural infusion, } \\
\text { intrathecal morphine injection, and transversus abdominis plane }\end{array}$ \\
Gynecologic surgeries & Quadratus lumborum, transversus abdominis plane, low thoracic dermatome para- \\
& vertebral block, thoracic or lumbar epidural infusion, and intrathecal morphine \\
& injection \\
\hline
\end{tabular}

- Rectus sheath block (RSB): Injection in the plane between the rectus abdominis muscle and posterior rectus sheath

- Ilioinguinal iliohypogastric nerves block: Injection in proximity to the ilioinguinal and iliohypogastric nerves, located within the plane between the internal oblique and transversus abdominis muscles in the lower quadrants of the anterior abdominal wall.

- Transversus abdominis plane (TAP) block: Injection in the plane between the internal oblique and transversus abdominis muscles

- Midaxillary transversus abdominis plane block: Injection in the plane between the internal oblique and transversus abdominis muscles at the midaxillary line

- Subcostal transversus abdominis plane block: Injection in the plane between the internal oblique and transversus abdominis muscles along the medial costal margin in the upper quadrants of the anterior abdominal wall [13], also referred by some as oblique subcostal transversus abdominis block (OSTAP)

- Anterior quadratus lumborum block (Anterior QLB): Injection in the plane between quadratus lumborum and psoas major muscles. This technique was previously referred to as the transmuscular quadratus lumborum (transmuscular QL) block as the block needle must pass through the muscle to inject on the anterior surface.

- Lateral quadratus lumborum block (Lateral QLB): Injection in the plane between the aponeuroses of internal oblique and transversus abdominis muscles at the lateral border of the quadratus lumborum muscle. This technique was previously referred to as the Quadratus Lumborum Type 1 (QL 1) block.

- Posterior quadratus lumborum block (Posterior QLB): Injection in the plane between the quadratus lumborum and erector spinae muscles, on the posterior surface of quadratus lumborum muscle. This technique was previously referred to as the quadratus lumborum type 2 (QL 2) block, denoting its position as the second QL block to be described in the literature.

\section{Medications}

Local anesthetics are the primary drugs injected when performing regional anesthesia. Various adjuvants have been described including epinephrine, clonidine, dexmedetomidine, tramadol, buprenorphine, and dexamethasone. These adjuvants may prolong the duration or increase the density of the induced block. However, it is important to note that many of the commonly used adjuvants for peripheral nerve blocks are not approved by the US Food and Drug Administration for perineural injection [38, 39]. As described above, opioid receptors in the spinal cord allow for epidurally and intrathecally administered fentanyl and morphine to exert a local effect, decreasing the need for systemically administered opioids. Results of studies comparing the addition of opioids to peripheral nerve blocks have been mixed with some finding benefit to perineural administration of opioids and others no benefit.

Lidocaine, ropivacaine, and bupivacaine are the most researched and utilized local anesthetics for peripheral and neuraxial blocks. This is due to their availability, pharmacodynamics, and pharmacokinetics. For abdominal plane blocks, a larger volume of more dilute local anesthetic is used compared to conventional peripheral nerve blocks or neuraxial techniques. This is due to the need for the local anesthetic solution to "hydro-dissect" the targeted plane and anesthetize the nerves running within.

For specific blocks, the optimal doses of the local anesthetics have been evaluated [2]. Fu et al. determined the dose of ropivacaine combined with butorphanol that is effective in 50\% (ED50) and 95\% (ED95) of subjects for successful pain-free ultrasound-guided RSB in single-incision laparoscopic cholecystectomy (SILC). They concluded that as part of a multimodal analgesia strategy, a dose of $0.719 \mathrm{mg} / \mathrm{kg}$ of ropivacaine provides successful RSB under ultrasound guidance in $50 \%$ of the patients who undergo SILC. A dose of $0.967 \mathrm{mg} / \mathrm{kg}$ would be successful in $95 \%$ of patients. However, for most peripheral plane and nerve blocks, the optimal dosing of local anesthetics has not been determined. 


\section{Timing of the Regional Anesthetic}

As peripheral blocks are usually performed with a single injection, as opposed to continuous infusion via an indwelling catheter (see below), correctly timing the nerve block is important to optimize the analgesic effect. Timing of blocks has been researched as well, focusing on the potential effect of "preemptive anesthesia and analgesia" to the pharmacokinetics of the local anesthetics injected [40]. Operating room efficiency must also be considered, weighing the block performance time against the improvement in recovery time and patient satisfaction associated with regional anesthetics. Surgeons have also come up with their own solutions such as injection of local anesthetic at the trocar site or the performance of TAP blocks under laparoscope visualization [41].

\section{Continuous Infusion Blocks}

Nerve blocks performed as a single injection of local anesthetic, with or without additives, have a duration that is measured in hours. While the most severe pain following laparoscopic surgery is in the immediate postoperative period, there is often pain that persists for days after surgery [11]. Continuous peripheral nerve blocks (CPNBs) utilize a catheter that is inserted-usually under ultrasound guidance-either adjacent to a peripheral nerve or into the plane targeted for an abdominal plane block [42, 43]. Local anesthetic may be infused for several days via this catheter, resulting in a peripheral block whose duration is measured in days, rather than hours. Patients at risk for prolonged pain (e.g., chronic opioid users, undergoing larger procedures, surgical drains left in place) may benefit from continuous infusions as opposed to single injection blocks. For abdominal plane blocks, relatively large, automated boluses may allow for more spread of local anesthetic than traditional continuous infusions of local anesthetic $[44,45]$.

\section{Outcomes}

Ultimately, the effect of regional anesthetics on patient outcomes is of paramount importance. Common outcomes in the regional anesthesia literature include pain at rest and with movement, time to discharge, opioid consumption in the first $48 \mathrm{~h}$ after surgery, patient satisfaction, incidence of nausea and vomiting, and patient readmission.

\section{Transversus Abdominis Plane Block}

Wu et al. compared the effect of an ultrasound-guided TAP block and rectus sheath block combination, and ultrasound-guided posterior TAP block combined with the local anesthetic infiltration (LAI) and LAI alone on pain relief after laparoscopic cholecystectomy (LC) in hundred eighty patients [46]. Patient satisfaction was assessed by The Global Satisfaction Score (GSS) for analgesia within $48 \mathrm{~h}$. The group found that ultrasound-guided peripheral nerve blocks of the abdominal wall can significantly relieve postoperative pain in patients undergoing laparoscopic cholecystectomy. However, in the study, patients receiving LAI expressed more satisfaction than patients in whom other methods were used. A 2018 meta-analysis and a 2020 systematic review and meta-analysis on TAP blocks for laparoscopic rectal surgery $[8,47]$ showed that TAP blocks can lead to a lower pain score at rest within the first $6 \mathrm{~h}$ and reduced opioid consumption within the first $24 \mathrm{~h}$, compared to no regional anesthetic pain regimens. Although TAP blocks may be associated with a reduced time to first bowel movement, patients receiving TAP blocks may not experience a shorter length of hospital stay (LOS) or a decrease in the incidence of nausea and vomiting. It is important again to mention that (LOS) is hard to measure as there are internal practices that affect patient discharge including surgeon's preferences, social issues and, the potential need for additional rehabilitation. In terms of nausea and vomiting again, the definitions vary by place as well as how the data is recorded in the medical record [48].

Selcuk et al. [49] studied the effect of the effects of preemptive and pre-closure analgesia on postoperative pain intensity in patients undergoing different levels of laparoscopic gynecological surgery. He found that the preclosure analgesia improved postoperative pain intensity during its half-life in both levels of surgery, which differed in terms of extent of visceral peritoneal dissection, operation time, and degree of manipulation. According to his results pain after laparoscopic gynecological surgery is mainly related to the parietal component.

Oblique subcostal TAP block (OSTAP) $[50,51]$ has been compared against mid-axillary TAP block and intravenous multimodal analgesia after laparoscopic cholecystectomy (LC). It has been found that OSTAP block has been more effective than the other 2 interventions previously mentioned in reducing postoperative pain scores and improving post-operative respiratory function. Ramkiran et al. [52] studied the usefulness of a rectus sheath block-OSTAP block combination, OSTAP block alone, and conventional port site infiltration in improving postoperative pain after LC. In this study, pain scores 
were significantly lower in the combined block group at the second postoperative hour. In addition, opioid consumption in the postoperative $24 \mathrm{~h}$ was significantly lower in the combined block group. It is important to notice that OSTAP does not reliably cover the lateral and posterior abdominal walls and that explains why study results are mixed and not all patients benefit from it. There is also a technical component to this block and has some provider-to-provider variability.

\section{Rectus Sheath Block}

The rectus sheath block has been considered as well for some laparoscopic procedures that required a trocar insertion above the T-10 dermatome. A very recent meta-analysis where nine trials and 698 patients were included [47] evaluated the efficacy and safety of RSB in adults undergoing laparoscopic surgery. RSB was associated with significantly lower rest pain scores at $0-2 \mathrm{~h}$ postoperatively than control. Also, RSB significantly reduced pain scores at rest at $10-12 \mathrm{~h}$ postoperatively and on movement at $0-2 \mathrm{~h}$ postoperatively, 24-h opioid consumption, and opioid-related side effects. Preoperative RSB provided better pain control compared with postoperative block administration. In the setting of laparoscopic surgery, RSB seems to improve pain control for up to $12 \mathrm{~h}$ postoperatively and reduce opioid consumption, without major reported adverse events. The fact that the block was done preoperatively may help explain the effect of the block in the first $12 \mathrm{~h}$. It is hard again to prove an association with pre-emptive analgesia.

\section{Quadratus Lumborum Block}

The quadratum lumborum block (QLB) has received more attention lately as there have been descriptions of the medication spreading into the paravertebral space. A recent metaanalysis [53] studied the duration of analgesia after posterior and lateral TAPB for lower abdominal surgery.

It included 12 trials comprising 641 patients. Four trials examined the posterior technique, and 8 trials examined the lateral technique. The posterior TAPB was found to produce more sustained analgesia than the lateral TAPB. As explained above, coverage for dermatome levels T7 to T12 was obtained by the QLB, whereas TAPB covered T10 to T12 dermatomes. Deng et al. [54] compared the posterior quadratus lumborum block (QLB) method with transversus abdominis plane block (TAPB) for postoperative pain management in patients undergoing laparoscopic colorectal surgery. In this study, seventyfour patients scheduled for laparoscopic colorectal surgery were randomly assigned to receive post-operative bilateral ultrasound-guided single-dose of QLB or TAPB with $20 \mathrm{ml}$ of $0.375 \%$ ropivacaine. The QLB group used significantly less sufentanil than TAPB group at 24 and $48 \mathrm{~h}$, but not at $6 \mathrm{~h}$.

\section{Erector Spinae Plane Block}

On the new techniques, the erector spinae block (ESP) has been the latest to be studied in patients undergoing laparoscopic cholecystectomy. Altiparmak et al. [18 ] compared the use of ESP versus subcostal TAP for this surgical procedure. In his study, he found that ultrasound-guided ESP block reduced postoperative tramadol consumption and pain scores more effectively than oblique subcostal transversus abdominis block (OSTAP) after laparoscopic cholecystectomy surgery. OSTAP covers dermatomes T6-T10, while ESP has been shown to be effective at covering both somatic and visceral pain. One of the theories that have been postulated as a reason for this is that in cadaveric studies, an injection of local anesthetic solution at the low thoracic level may extend anteriorly and enter the thoracic paravertebral space. A recent report of 3 cases show that it is possible for the local anesthetic to reach the rami communicantes that transmit fibers to and from the sympathetic ganglia and block visceral pain while also blocking somatic pain by blocking the ventral and dorsal rami of spinal nerves [55]. There is also spread of local anesthetic to the epidural, neural foraminal, and intercostal areas improving pain coverage.

The combination of techniques has also been studied. Liang et al. investigated the analgesic effects of US-guided posterior TAPB with RSB on postoperative pain following laparoscopy-assisted radical resection of early-stage rectal cancer. In this study, seventy-eight adults scheduled for laparoscopy-assisted radical resection of rectal cancer were randomized to 3 groups: US guided bilateral posterior TAPB (40 $\mathrm{mL} 0.33 \%$ ropivacaine) with $\mathrm{RSB}$ (20 mL $0.33 \%$ ropivacaine), US-guided bilateral posterior TAPB alone, and a Control Group. The group receiving the combined blocks had significantly lower postoperative use of PCIA and rescue analgesic than in the other two groups. This can be explained by the anatomy and coverage of dermatomes provided by each technique. As many other have pointed out a single technique may not be enough to cover for all incisions and dermatomes, something that is better accomplished by neuraxial blocks. That is the case of liver resection where TAP block reduces the use of fentanyl by $20 \%$, while that RSB combined with TAPB reduces the use of fentanyl by more than $60 \%$ [56].

\section{Robotic Surgery}

The area of robotic surgery is one that can be studied as a group when looking at local and regional anesthesia techniques. Shahalt and Lee [57] looked at the application of TAP blocks in laparoscopic urological surgery. They found 
that TAP block decreases postoperative pain and reduces opioid consumption without increasing complications in robot-assisted radical prostatectomy, minimally invasive renal surgery, and cystectomy.

\section{Complications}

As with any intervention, complications related to regional anesthesia are possible and should be weighed against the benefits of decreased pain, reduction in opioid consumption and related adverse effects, and potential for facilitating same-day discharge. Common complications associated with any regional anesthetic procedure are bleeding, infection, injury to surrounding structures, local anesthetic toxicity, and allergic reactions. Certain techniques are associated with specific risks (e.g., pneumothorax following paravertebral block and spinal hematoma following epidural or intrathecal block) and known side effects (e.g., hypotension resulting from the sympathectomy produced by neuraxial anesthesia). Peripheral blocks may be associated with a reduction in risk; however, serious complications can arise with these blocks as well [58]. Care should be taken to minimize risk to patients, including: utilization of proper sterile technique, adherence to the American Society of Regional Anesthesia and Pain Medicine Evidence-Based Guidelines for regional anesthesia in patients receiving antithrombotic or thrombolytic therapy, and ultrasound guidance when feasible [59].

\section{Conclusion}

As the trend toward minimally invasive surgical procedures continues, there will be increasing demand for anesthetic options that decrease systemic opioid requirements, hasten return of bowel function, and facilitate same-day discharge. Conventional regional anesthetic options, such as thoracic epidural infusions and intrathecal opioid administration, remain excellent options for decreasing postoperative pain, opioid consumption, and length of stay following major colorectal and gynecologic laparoscopic surgeries. These neuraxial techniques, unfortunately, require hospital admission. Paravertebral and peripheral blocks may offer many of the benefits of epidural or intrathecal blocks, while allowing for same-day hospital discharge or performing laparoscopic surgery at ambulatory surgery centers. Research is ongoing to determine the optimal peripheral block techniques, type, and dose of local anesthetic, whether and how to utilize continuous block techniques in the ambulatory setting, and timing of single injection nerve blocks. The regional anesthesiologist is now equipped with a diverse armamentarium for managing pain after laparoscopic surgery, and the type of surgery, as well as the setting, should determine which blocks he or she uses for a given patient.
Funding John Finneran: The University of California has received funding and product for Dr. Finneran's research from Epimed (Farmers Branch, TX), InfuTronix (Natick, MA), and SPR Therapeutics (Cleveland, $\mathrm{OH}$ ).

\section{Declarations}

Conflict of Interest Alvaro Andres Macias: I report no conflict of interests and have no disclosures.

Human and Animal Rights and Informed Consent This article does not contain any studies with human or animal subjects performed by any of the authors.

\section{References}

Papers of particular interest, published recently, have been highlighted as:

- Of importance

1. Rosero EB, Joshi GP. Hospital readmission after ambulatory laparoscopic cholecystectomy: incidence and predictors. J Surg Res [Internet]. 2017;219:108-15. Available from: https://doi. org/10.1016/j.jss.2017.05.071. This paper addresses hospital readmission after ambulatory laparoscopic cholecystectomy by using big data.

2. Fu H, Fu Y, Xu X, Gao Y. Ultrasound-guided rectus sheath block combined with butorphanol for single-incision laparoscopic cholecystectomy: what is the optimal dose of ropivacaine? J Pain Res. 2020;13:2609-15.

3. Pirrera B, Alagna V, Lucchi A, Berti P, Gabbianelli C, Martorelli $\mathrm{G}$, et al. Transversus abdominis plane (TAP) block versus thoracic epidural analgesia (TEA) in laparoscopic colon surgery in the ERAS program. Surg Endosc. 2018;32(1):376-82.

4. Maloney C, Kallis M, El-Shafy IA, Lipskar AM, Hagen J, Kars M. Ultrasound-guided bilateral rectus sheath block vs. conventional local analgesia in single port laparoscopic appendectomy for children with nonperforated appendicitis. J Pediatr Surg [Internet]. 2018;53(3):431-6. Available from: https://doi.org/ 10.1016/j.jpedsurg.2017.05.027.

5. Steiner CA, Zeynal K, Moore BJ, Imshaug MC, Pickens G. Surgeries in hospital-based ambulatory surgery and hospital inpatient settings [Internet]. H-CUP. 2020 [cited 2021 Oct 8]. Available from: https://www.hcup-us.ahrq.gov/reports/statbriefs/ sb223-Ambulatory-Inpatient-Surgeries-2014.jsp.

6. Gignoux B, Blanchet MC, Lanz T, Vulliez A, Saffarini M, Bothorel $\mathrm{H}$, et al. Should ambulatory appendectomy become the standard treatment for acute appendicitis? World J Emerg Surg. 2018;13(1):1-8.

7. Chamberlain RS, Sakpal SV. A Comprehensive review of singleincision laparoscopic surgery (SILS) and natural orifice transluminal endoscopic surgery (NOTES) techniques for cholecystectomy. J Gastrointest Surg. 2009;13(9):1733-40.

8. Hain E, Maggiori L, Prost à la Denise J, Panis Y. Transversus abdominis plane (TAP) block in laparoscopic colorectal surgery improves postoperative pain management: a meta-analysis. Color Dis. 2018;20(4):279-87.

9. Nyerges A. Pain mechanisms in laparoscopic surgery. Semin Laparosc Surg. 1994;1(4):215-8.

10. Naja ZM, El-Rajab M, Ziade F, Al-Tannir M, Itani T. Preoperative vs. postoperative bilateral paravertebral blocks for 
laparoscopic cholecystectomy: a prospective randomized clinical trial. Pain Pract. 2011;11(6):509-15.

11. Ekstein P, Szold A, Sagie B, Werbin N, Klausner JM, Weinbroum AA. Laparoscopic surgery may be associated with severe pain and high analgesia requirements in the immediate postoperative period. Ann Surg. 2006;243(1):41-6.

12. Mouton WG, Bessell JR, Otten KT, Maddern GJ. Pain after laparoscopy. Surg Endosc. 1999;13(5):445-8.

13. Hebbard PD, Barrington MJ, Vasey C. Ultrasound-guided continuous oblique subcostal transversus abdominis plane blockade: description of anatomy and clinical technique. Reg Anesth Pain Med. 2010;35(5):436-41.

14. El-Boghdadly K, Wolmarans M, Stengel AD, Albrecht E, Chin KJ, Elsharkawy H, et al. Standardizing nomenclature in regional anesthesia: an ASRA-ESRA Delphi consensus study of abdominal wall, paraspinal, and chest wall blocks. Reg Anesth Pain Med. 2021;46(7):571-80. This paper addresses the need for standardization of the current abdominal wall, paraspinal and chest wall regional techniques. This allows better research and clinical application of the techniques.

15. Elsharkawy H, El-Boghdadly K, Barrington M. Quadratus lumborum block. Anesthesiology. 2019;130(2):322-35.

16. Drake, Richard. Vogl, W. Mitchell A. Gray's anatomy for students. In: Gray's anatomy for students. 4th ed.

17. Vleeming A, Schuenke MD, Danneels L, Willard FH. The functional coupling of the deep abdominal and paraspinal muscles: the effects of simulated paraspinal muscle contraction on force transfer to the middle and posterior layer of the thoracolumbar fascia. J Anat. 2014;225(4):447-62.

18. Altıparmak B, Korkmaz Toker M, Uysal AI, Kuşçu Y, Gümüş Demirbilek S. Ultrasound-guided erector spinae plane block versus oblique subcostal transversus abdominis plane block for postoperative analgesia of adult patients undergoing laparoscopic cholecystectomy: randomized, controlled trial. J Clin Anesth [Internet]. 2019;57(December 2018):31-6. Available from: https://doi.org/10.1016/j.jclinane.2019.03.012. This paper is one of the first ones to address the effectiveness of erector spinae plane block for ambulatory laparoscopic surgery.

19. Lim Y, Ocampo CE, Sia AT. A comparison of duration of analgesia of intrathecal $2.5 \mathrm{mg}$ of bupivacaine, ropivacaine, and levobupivacaine in combined spinal epidural analgesia for patients in labor. Anesth Analg. 2004;98(1):235-9.

20. Rasmussen SB, Saied NN, Bowens C, Mercaldo ND, Schildcrout JS, Malchow RJ. Duration of upper and lower extremity peripheral nerve blockade is prolonged with dexamethasone when added to ropivacaine: a retrospective database analysis. Pain Med (United States). 2013;14(8):1239-47.

21. Erol DD, Yilmaz S, Polat C, Arikan Y. Efficacy of thoracic epidural analgesia for laparoscopic cholecystectomy. Adv Ther. 2008;25(1):45-52.

22. Zingg U, Miskovic D, Hamel CT, Erni L, Oertli D, Metzger U. Influence of thoracic epidural analgesia on postoperative pain relief and ileus after laparoscopic colorectal resection: BBBenefit with epidural analgesia. Surg Endosc. 2009;23(2):276-82.

23. Taqi A, Hong X, Mistraletti G, Stein B, Charlebois P, Carli F. Thoracic epidural analgesia facilitates the restoration of bowel function and dietary intake in patients undergoing laparoscopic colon resection using a traditional, nonaccelerated, perioperative care program. Surg Endosc Other Interv Tech. 2007;21(2):247-52.

24. Kim MK, Baek CW, Kang H, Choi GJ, Park YH, Yang SY, et al. Comparison of emergence after deep extubation using desflurane or desflurane with remifentanil in patients undergoing general anesthesia: a randomized trial. J Clin Anesth. 2016.
25. Yaksh T. The physiology and pharmacology of spinal opiates. Middle East J Anesthesiol. 1990;10(4):413-22.

26. Karakaya D, Büyükgöz F, Barş S, Güldouş F, Tür A. Addition of fentanyl to bupivacaine prolongs anesthesia and analgesia in axillary brachial plexus block. Reg Anesth Pain Med. 2001;26(5):434-8.

27. Candido KD, Winnie AP, Ghaleb AH, Fattouh MW, Franco CD. Buprenorphine added to the local anesthetic for axillary brachial plexus block prolongs postoperative analgesia. Reg Anesth Pain Med. 2002;27(2):162-7.

28. Fanelli G, Casati A, Magistris L, Berti M, Albertin M Scarioni A, Torri G. Fentanyl does not improve the nerve block characteristics of axillary brachial plexus anaesthesia performed with ropivacaine. Acta Anaesthesiol Scand. 2001;45(5):590-4.

29. Kong SK, Onsiong SMK, Chiu WKY, Li MKW. Use of intrathecal morphine for postoperative pain relief after elective laparoscopic colorectal surgery. Anaesthesia. 2002;57(12):1168-73.

30. Motamed C, Bouaziz H, Franco D, Benhamou D. Analgesic effect of low-dose intrathecal morphine and bupivacaine in laparoscopic cholecystectomy. Anaesthesia. 2000;55(2):118-24.

31. Kang RA, Chin KJ, Gwak MS, Kim GS, Choi SJ, Kim JM, et al. Bilateral single-injection erector spinae plane block versus intrathecal morphine for postoperative analgesia in living donor laparoscopic hepatectomy: a randomized non-inferiority trial. Reg Anesth Pain Med. 2019;44(12):1059-65.

32. Sultan P, Gutierrez MC, Carvalho B. Neuraxial morphine and respiratory depression: Finding the right balance. Drugs. 2011;71(14):1807-19.

33. Aydin G, Aydin O. The efficacy of ultrasound-guided paravertebral block in laparoscopic cholecystectomy. Med. 2018;54(5):2-9.

34. Naja MZ, Ziade MF, Lönnqvist PA. General anaesthesia combined with bilateral paravertebral blockade (T5-6) vs. general anaesthesia for laparoscopic cholecystectomy: a prospective, randomized clinical trial. Vol. 21, European Journal of Anaesthesiology. 2004. p. 489-95.

35. Naja ZM, El-Rajab M, Al-Tannir MA, Ziade FM, Tayara $\mathrm{K}$, Younes F, et al. Thoracic paravertebral block: influence of the number of injections. Reg Anesth Pain Med. 2006;31(3):196-201.

36. Schneider LF, Albornoz CR, Huang J, Cordeiro PG. Incidence of pneumothorax during tissue expander-implant reconstruction and algorithm for intraoperative management. Ann Plast Surg. 2014;73(3):279-81.

37. Niesen AD, Jacob AK, Law LA, Sviggum HP, Johnson RL. Complication rate of ultrasound-guided paravertebral block for breast surgery. Reg Anesth Pain Med. 2020;45(10):813-7.

38. Krishna Prasad GV, Khanna S, Jaishree S. Review of adjuvants to local anesthetics in peripheral nerve blocks: current and future trends. Saudi J Anaesth. 2020;14(1):77-84.

39. Choi S, Rodseth R, McCartney CJL. Effects of dexamethasone as a local anaesthetic adjuvant for brachial plexus block: a systematic review and meta-analysis of randomized trials. Br J Anaesth [Internet]. 2014;112(3):427-39. Available from: https://doi.org/ 10.1093/bja/aet417.

40. Oh TK, Lee SJ, Do SH, Song IA. Transversus abdominis plane block using a short-acting local anesthetic for postoperative pain after laparoscopic colorectal surgery: a systematic review and meta-analysis. Surg Endosc. 2018;32(2):545-52.

41. Ortiz J, Suliburk JW, Wu K, Bailard NS, Mason C, Minard $\mathrm{CG}$, et al. Bilateral transversus abdominis plane block does not decrease postoperative pain after laparoscopic cholecystectomy when compared with local anesthetic infiltration of trocar insertion sites. Reg Anesth Pain Med. 2012;37(2):188-92.

42. Ilfeld BM. Continuous peripheral nerve blocks: a review of the published evidence. Anesth Analg. 2011;113(4):904-25. 
43. Ilfeld BM. Continuous peripheral nerve blocks: an update of the published evidence and comparison with novel, alternative analgesic modalities. Anesth Analg. 2017;124(1):308-35.

44. Finneran JJ, Alexander B, Bechis SK, Sur RL, Ilfeld BM. Continuous erector spinae plane blocks with automated boluses for analgesia following percutaneous nephrolithotomy. Korean J Anesthesiol. 2021;74(2):178-80.

45. Chen L, Wu Y, Cai Y, Ye Y, Li L, Xia Y, et al. Comparison of programmed intermittent bolus infusion and continuous infusion for postoperative patient-controlled analgesia with thoracic paravertebral block catheter: a randomized, double-blind, controlled trial. Reg Anesth Pain Med. 2019;44(2):240-5.

46. Wu L, Wu L, Sun H, Dong C, Yu J. Corrigendum: effect of ultrasound-guided peripheral nerve blocks of the abdominal wall on pain relief after laparoscopic cholecystectomy (J Pain Res. 2019;12:1433-1439). J Pain Res. 2020;13:2169.

47. Hamid HKS, Ahmed AY, Alhamo MA, Davis GN. Efficacy and safety profile of rectus sheath block in adult laparoscopic surgery: a meta-analysis. J Surg Res [Internet]. 2021;261:10-7. Available from: https://doi.org/10.1016/j.jss.2020.12.003.

48. Wu RC, Jensen CC, Douaiher J, Madoff RD, Kwaan MR. Transversus abdominis plane block in laparoscopic colorectal surgery: a systematic review. Dis Colon Rectum. 2019;62(10):1248-55.

49. Selcuk S, Api M, Polat M, Arinkan A, Aksoy B, Akca T, et al. Effectiveness of local anesthetic on postoperative pain in different levels of laparoscopic gynecological surgery. Arch Gynecol Obstet. 2016;293(6):1279-85.

50. Oksar M, Koyuncu O, Turhanoglu S, Temiz M, Oran MC. Transversus abdominis plane block as a component of multimodal analgesia for laparoscopic cholecystectomy. J Clin Anesth [Internet]. 2016;34:72-8. Available from: https://doi.org/10.1016/j. jclinane.2016.03.033.

51. Basaran B, Basaran A, Kozanhan B, Kasdogan E, Eryilmaz MA, Ozmen S. Analgesia and respiratory function after laparoscopic cholecystectomy in patients receiving ultrasound-guided bilateral oblique subcostal transversus abdominis plane block: a randomized double-blind study. Med Sci Monit. 2015;21:1304-12.

52. Ramkiran S, Jacob M, Honwad M, Vivekanand D, Krishnakumar M, Patrikar S. Ultrasound-guided combined fascial plane blocks as an intervention for pain management after laparoscopic cholecystectomy: a randomized control study. Anesth Essays Res. 2018;12(1):16.

53. Abdallah FW, Laffey JG, Halpern SH, Brull R. Duration of analgesic effectiveness after the posterior and lateral transversus abdominis plane block techniques for transverse lower abdominal incisions: a meta-analysis. Br J Anaesth [Internet]. 2013;111(5):721-35. Available from: https://doi.org/10.1093/ bja/aet214.

54. Deng W, Long X, Li M, Li C, Guo L, Xu G, et al. Quadratus lumborum block versus transversus abdominis plane block for postoperative pain management after laparoscopic colorectal surgery: a randomized controlled trial. Med (United States). 2019;98(52):1-5.

55. Chin KJ, Malhas L, Perlas A. The erector spinae plane block provides visceral abdominal analgesia in bariatric surgery a report of 3 cases. Reg Anesth Pain Med. 2017;42(3):372-6.

56. Yassen K, Lotfy M, Miligi A, Sallam A, Hegazi EARAM. Patient-controlled analgesia with and without transverse abdominis plane and rectus sheath space block in cirrhotic patients undergoing liver resection. J Anaesthesiol Clin Pharmacol. 2019;35(1):58-64.

57. Shahait M, Lee DI. Application of TAP block in laparoscopic urological surgery: current status and future directions. Curr Urol Rep. 2019;20(5):13-6.

58. Ueshima H. Pneumothorax after the erector spinae plane block. J Clin Anesth [Internet]. 2018;48(April):12. Available from: https://doi.org/10.1016/j.jclinane.2018.04.009.

59. Horlocker TT, Vandermeuelen E, Kopp SL, Gogarten W, Leffert LR, Benzon HT. Regional anesthesia in the patient receiving antithrombotic or thrombolytic therapy: American Society of Regional Anesthesia and Pain Medicin Evidence-Based Guidelines (Fourth Edition). Vol. 43, Regional Anesthesia and Pain Medicine. 2018;263-309.

Publisher's Note Springer Nature remains neutral with regard to jurisdictional claims in published maps and institutional affiliations. 